\title{
Wirksamkeit von weiblichen Geschlechtshormonen und P-armer und Ca-reicher Diät auf die ${ }^{90} \mathrm{Sr}$-Dekorporation
}

\author{
Irmhild Steinbach \\ Heiligenberg-Institut, Heiligenberg/Baden, Abteilung für Radioisotopenforschung
}

(Leiter: Prof. Dr. Dr. h. c. H. LangendorfF)

(Z. Naturforsch. 23 b, 820-824 [1968]; eingegangen am 8. Juni 1967)

\begin{abstract}
In zwei getrennt durchgeführten Versuchen mit Rattenmännchen und kastrierten Rattenmännchen wurde der Einfluß von P-armer und Ca-reicher Diät, von Östrogen und von Progesteron auf den ${ }^{90} \mathrm{Sr}$-Stoffwechsel untersucht.

P-arme und Ca-reiche Diät erniedrigt den ${ }^{90} \mathrm{Sr}$-Gehalt. Östrogenverabreichung führt zu einer erhöhten ${ }^{90} \mathrm{Sr}$-Retention. Beim Progesteron konnte kein Einfluß auf die ${ }^{90} \mathrm{Sr}$-Ausscheidung nachgewiesen werden. Bei kombinierter Verabreichung addieren sich die Wirkungen der 3 Behandlungsarten.
\end{abstract}

Eine bekannte Möglichkeit, die ${ }^{90} \mathrm{Sr}$-Ausscheidung zu erhöhen, besteht in der Verabreichung einer P. armen und Ca-reichen Diät ${ }^{3,4,6,8}$.

Gestation und Laktation bei Ratten steigern ebenfalls die ${ }^{90} \mathrm{Sr}$-Ausscheidung ${ }^{2,5}$. Diese Wirkung der Gestation und Laktation auf die ${ }^{90} \mathrm{Sr}$-Dekorporation wird wahrscheinlich durch Hormone gesteuert.

In zwei getrennten Experimenten wurde bei männlichen Ratten und kastrierten männlichen Ratten die Wirksamkeit weiblicher Geschlechtshormone und Parmer und Ca-reicher Diät, deren Verabreichung einzeln und in den möglichen Kombinationen erfolgte, auf die ${ }^{90} \mathrm{Sr}$-Dekorporation untersucht.

\section{Methodik}

\section{Versuch I}

48 männliche Ratten des Heiligenberg-Stammes wurden in 8 Behandlungsgruppen zu je 6 Tieren zufällig aufgeteilt. Die 6 Wochen alten Tiere hatten ein Durchschnittsgewicht von $140 \mathrm{Gramm}$. Eine praktisch trägerfreie ${ }^{90}-\mathrm{SrCl}_{2}$-Lösung wurde intravenös injiziert. Die verabreichte Aktivität betrug $10 \mu \mathrm{Ci}$ pro Tier. 24 Stdn. nach der ${ }^{90} \mathrm{Sr}$-Injektion begann die Behandlung der Tiere mit Normalfutter, P-armer und Ca-reicher Diät, im folgenden nur noch Diät genannt, mit Östrogen und Progesteron und wurde 3 Wochen p.i. beendet.

Das Normalfutter hat einen Calcium- und Phosphorgehalt von $13,7 \mathrm{~g}$ bzw. 9,3 g pro $1000 \mathrm{~g}$ Futter. Die Diät hat einen Ca- und P-Gehalt von $31,1 \mathrm{~g}$ bzw. $2,1 \mathrm{~g}$ pro 1000 g Futter. Normalfutter und Diät wurden ad

1 R. Ammon u. W. Dirscherl, Fermente-Hormone-Vitamine. Bd. II, Hormone, 3. Auflage. Georg Thieme-Verlag, Stuttgart 1960.

2 W. E. Kollmer u. H. Kriegel, Nature [London] 200, 4902, 187 [1963 a] ; 205, 4967, 196 [1965] ; Int. J. Rad. Biol. 7, 333 [1963 b] ; 9, 4, 369 [1965]. lib. verabreicht. Als Östrogen wurde Progynon in Sesamöl gelöst und i.m. injiziert. Die dem Tier täglich verabreichte Menge betrug 0,02 mg Progynon in $0,1 \mathrm{ml}$ Sesamöl. Als Progesteron wurde Proluton in Sesamöl gelöst und i.m. injiziert. Die dem Tier täglich verabreichte Menge betrug $0,3 \mathrm{mg}$ Proluton in $0,1 \mathrm{ml}$ Sesamöl.

Die Aufteilung der Tiergruppen auf die verschiedenen Behandlungsverfahren geht aus Tab. 1 hervor.

6 Wochen nach der Injektion wurde nach vorausgegangener Gewichtsbestimmung der ${ }^{90} \mathrm{Sr}$-Gehalt der Tiere mit einem Ganzkörperzähler der Fa. Packard ermittelt. 14 Wochen p.i. wurden die Ratten abgetötet

\begin{aligned} & \hline Gruppe \\ & \hline I Normalfutter (= Kontrolle) \\ & II Diät \\ & III Östrogen und Normalfutter \\ & IV Progesteron und Normalfutter \\ & V Östrogen und Diät \\ & VI Progesteron und Diät \\ & VII Östrogen und Progesteron und Normalfutter \\ & VIII Östrogen und Progesteron und Diät \\ & \hline\end{aligned}

Tab. 1. Behandlungsgruppen der Versuche I und II.

und die Femora präpariert. Nach der Veraschung der Femora wurde das Aschegewicht bestimmt und nach den gebräuchlichen Methoden der ${ }^{90} \mathrm{Sr}$-Gehalt und die ${ }^{90} \mathrm{Sr}$-Konzentration des Femurs ermittelt.

\section{Versuch II}

32 männliche Ratten des Heiligenberg-Stammes wurden im Alter von 5 Wochen kastriert. 3 Wochen nach der Kastration wurden die Ratten in 8 Behandlungs-

3 K. Kostial, A. Lutkić, N. Gruden u. Vojvodić, Int. J. Rad. Biol. 6, 5, 431 [1963].

4 H. Kriegel, W. E. Kollmer u. E. Weber, Int. J. Rad. Biol. 7, 3, 289 [1963].

5 H. Kriegel u. G. K. Neumans, Atompraxis 2, 8 [1962]. 


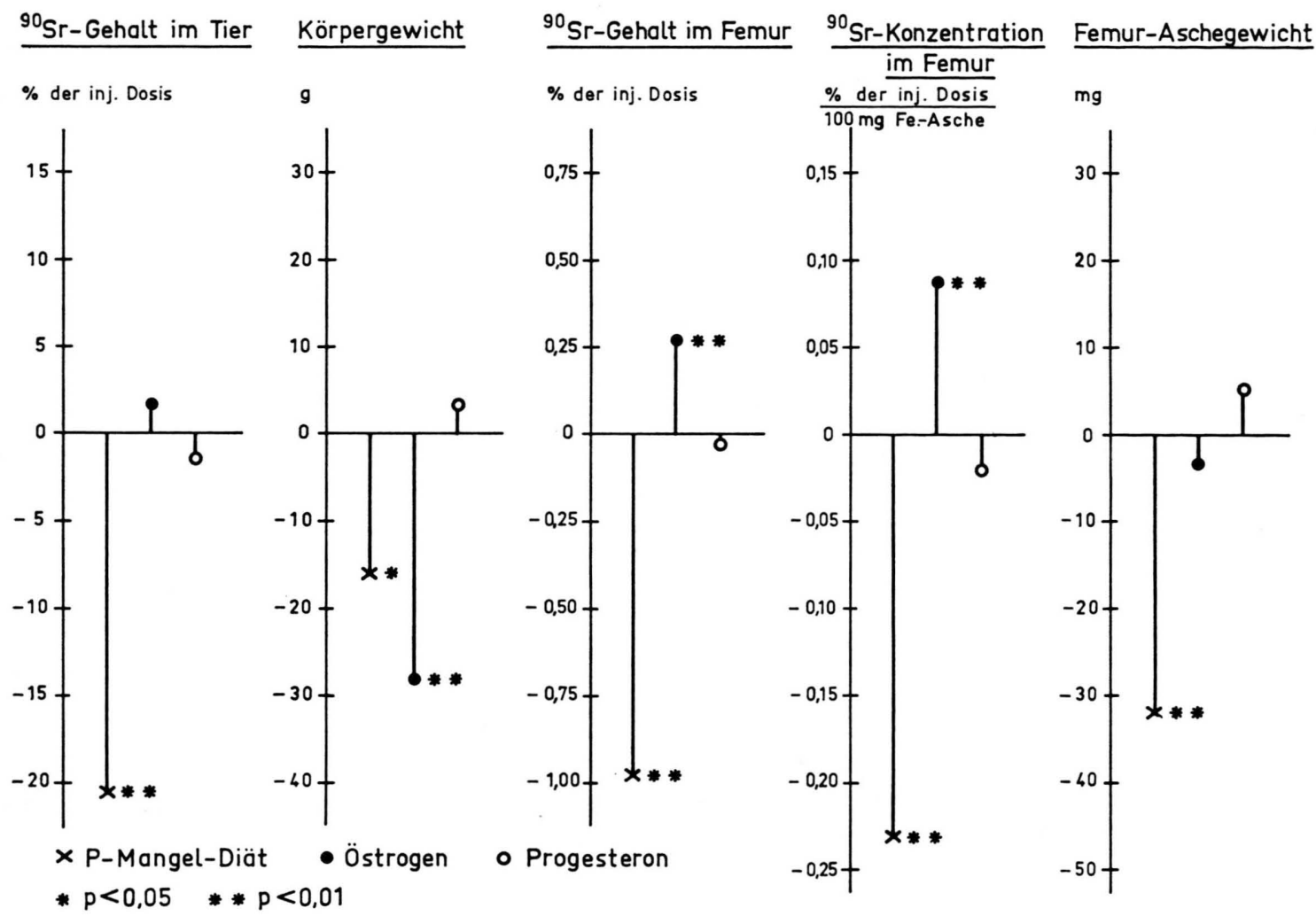

Abb. 1. Wirkung der Diät, des Östrogens und des Progesterons auf den ${ }^{90} \mathrm{Sr}$-Gehalt, das Körpergewicht und das Aschegewicht bei der männlichen Ratte.

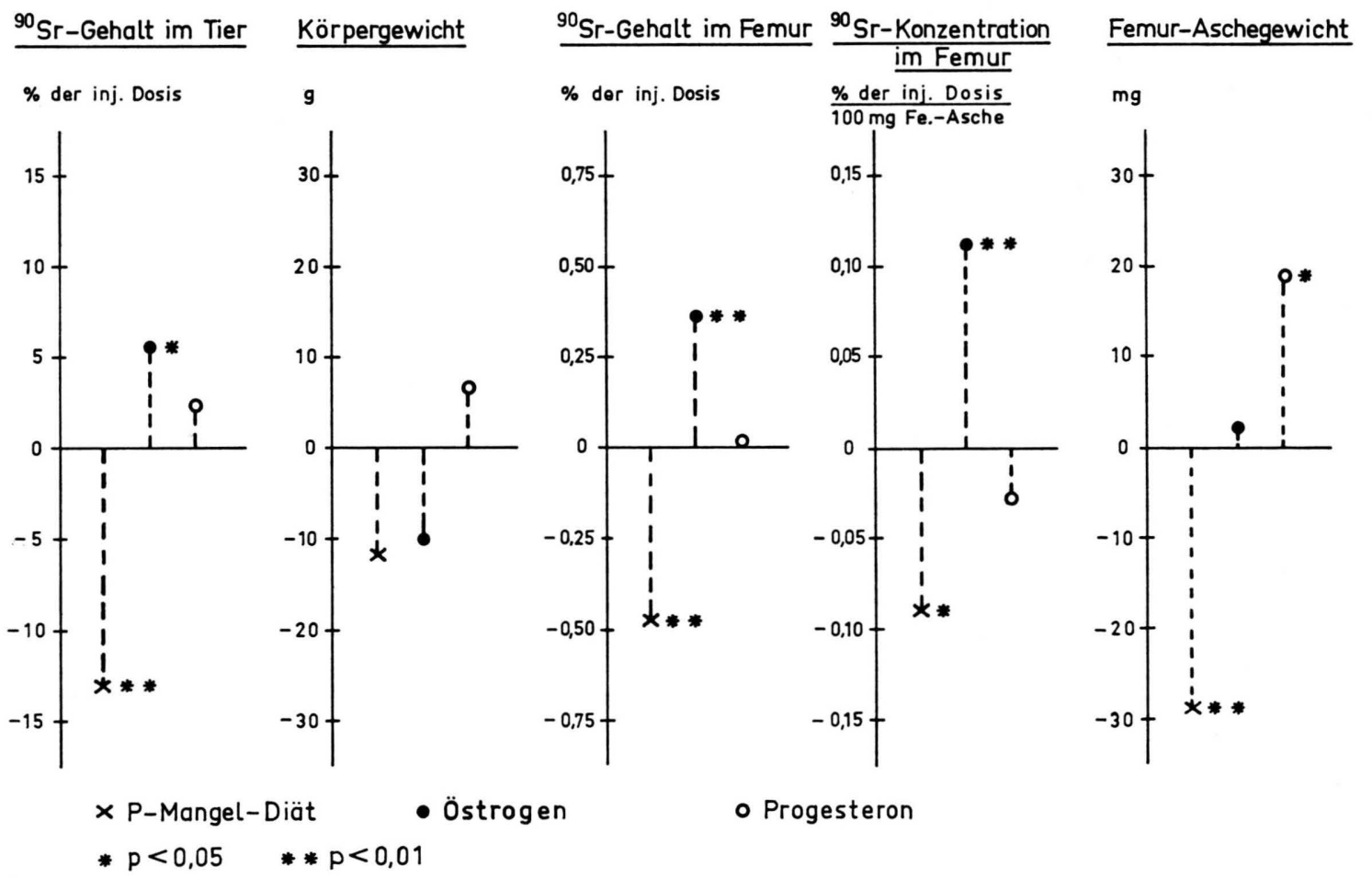

Abb. 2. Wirkung der Diät, des Östrogens und des Progesterons auf den ${ }^{90} \mathrm{Sr}$-Gehalt, das Körpergewicht und das Aschegewicht bei der kastrierten männlichen Ratte. 
gruppen mit je 4 Tieren aufgeteilt. Das Durchschnittsgewicht der Tiere betrug 200 Gramm. Die weitere Durchführung des Versuches entsprach der Methodik des vorausgegangenen Versuchs. Nur die Bestimmung des Femuraschegewichts, des ${ }^{90} \mathrm{Sr}-\mathrm{Gehaltes}$ und der ${ }^{90} \mathrm{Sr}$-Konzentration im Femur erfolgte bereits 8 Wochen p. i.

Die statistische Auswertung der Meßergebnisse des Versuches I und II erfolgte mit einer Varianzanalyse. Mittels orthogonaler Vergleiche wurden die Wirkungen der Diät, des Östrogens und des Progesterons auf die gemessenen Größen berechnet. Die Ergebnisse sind mit Angabe der Irrtumswahrscheinlichkeit in den Abbn. 1 und 2 dargestellt. Die Wirkungen sind in diesen Darstellungen absolute positive oder negative Abweichungen der Mittelwerte der behandelten Tiere von den Mittelwerten der Kontrolltiere.

\section{Ergebnisse}

\section{Versuch $I$}

In Tab. 2 sind die Untersuchungsergebnisse für die männlichen Tiere zusammengestellt. Die Zahlenangaben sind Mittelwerte, die aus jeweils 6 Einzelwerten errechnet wurden. Die Spalten der Tab. 2 geben die einzelnen Verfahren wieder und die Zeilen entsprechen den Meßgrößen. Dabei beziehen sich die ersten beiden Zeilen auf den ${ }^{90} \mathrm{Sr}$-Gehalt des ganzen Tieres, ausgedrückt in \% der injizierten Dosis und auf das Körpergewicht in Gramm. Diese Messungen wurden 6 Wochen nach der Injektion des ${ }^{90} \mathrm{Sr}$ durchgeführt. Die folgenden Zeilen geben in der gleichen Reihenfolge den ${ }^{90} \mathrm{Sr}$-Gehalt des Femurs (\% der injizierten Dosis), die ${ }^{90} \mathrm{Sr}-$ Konzentration des Femurs (\% der injizierten Dosis pro $100 \mathrm{mg}$ Femurasche) und das Aschegewicht des Femurs (mg) wieder. Die Bestimmungen dieser Größen wurden
14 Wochen nach der Injektion des ${ }^{90} \mathrm{Sr}$ vorgenommen.

Die Wirkungen der Diät, des Östrogens und des Progesterons sind in Abb. 1 dargestellt. Die Verabreichung der Diät führte zu einer Verminderung des ${ }^{90} \mathrm{Sr}-G e h a l t e s$ im Gesamttier um etwa $20 \%$ der injizierten Dosis. Das Körpergewicht lag im Vergleich zum Kontrolltier um $16 \mathrm{~g}$ niedriger. Die Abnahme des ${ }^{90} \mathrm{Sr}$-Gehaltes und der ${ }^{90} \mathrm{Sr}$-Konzentration im Femur betrugen 1\% der injizierten Dosis bzw. $0,23 \%$ der injizierten Dosis pro $100 \mathrm{mg}$ Femurasche. Der Einfluß auf das Femuraschegewicht bestand in einer Erniedrigung um 32 Milligramm.

Die geringe Erhöhung des ${ }^{90} \mathrm{Sr}-G e h a l t e s ~ i m ~ G a n z-$ tier nach Östrogengaben konnte nicht gesichert werden. Die Östrogenbehandlung verursachte eine Erniedrigung des Körpergewichts um 28 Gramm. Der ${ }^{90} \mathrm{Sr}$-Gehalt und die ${ }^{90} \mathrm{Sr}$-Konzentration des Femurs waren um $0,27 \%$ der injizierten Dosis und $0,05 \%$ der injizierten Dosis pro $100 \mathrm{mg}$ Femurasche erhöht. Eine Wirkung auf das Femuraschegewicht konnte nicht festgestellt werden.

Die Progesteronbehandlung zeigte keinen gesicherten Einfluß auf die untersuchten Größen. Nach kombinierter Anwendung der drei Behandlungsarten wurden keine Veränderungen der Einzelwirkungen beobachtet.

\section{Versuch II}

In Tab. 3 sind die Untersuchungsergebnisse für die männlichen Kastraten zusammengestellt. Die Zahlenangaben sind Mittelwerte, die aus jeweils 4 Einzelwerten errechnet wurden. Der ${ }^{90} \mathrm{Sr}$-Gehalt des ganzen Tieres und das Körpergewicht wurden 6 Wochen nach der ${ }^{90} \mathrm{Sr}$-Injektion gemessen. Die Be-

\begin{tabular}{|c|c|c|c|c|c|c|c|c|}
\hline & Kontrolle & Diät & Östrogen & Progesteron & $\begin{array}{l}\text { Östrogen } \\
\text { und Diät }\end{array}$ & $\begin{array}{l}\text { Progesteron } \\
\text { und Diät }\end{array}$ & $\begin{array}{l}\text { Östrogen } \\
\text { und } \\
\text { Progesteron }\end{array}$ & $\begin{array}{l}\text { Östrogen } \\
\text { und } \\
\text { Progesteron } \\
\text { und Diät }\end{array}$ \\
\hline $\begin{array}{l}{ }^{90} \text { Sr-Gehalt in } \% \mathrm{~d} . \\
\text { inj. Dosis/Tier }\end{array}$ & 75,81 & 50,47 & 77,35 & 73,38 & 55,63 & 53,51 & 73,95 & 53,61 \\
\hline Körpergewicht $[\mathrm{g}]$ & 262 & 250 & 235 & 276 & 221 & 242 & 233 & 231 \\
\hline $\begin{array}{l}{ }^{90} \text { Sr-Gehalt in \% d. } \\
\text { inj. Dosis/Femur }\end{array}$ & 2,52 & 1,48 & 2,71 & 2,47 & 1,83 & 1,47 & 2,72 & 1,78 \\
\hline $\begin{array}{l}{ }^{90} \mathrm{Sr}-\text { Konz. in } \% \mathrm{~d} . \\
\text { inj. Dosis } / 100 \mathrm{mg} \\
\text { Femurasche }\end{array}$ & 0,71 & 0,46 & 0,80 & 0,68 & 0,56 & 0,47 & 0,76 & 0,55 \\
\hline $\begin{array}{l}\text { Femuraschegewicht } \\
{[\mathrm{mg}]}\end{array}$ & 357 & 327 & 337 & 368 & 330 & 316 & 364 & 324 \\
\hline
\end{tabular}

Tab. 2. Untersuchungsergebnisse des Versuches I. 


\begin{tabular}{|c|c|c|c|c|c|c|c|c|}
\hline & Kontrolle & Diät & Östrogen & Progesteron & $\begin{array}{l}\text { Östrogen } \\
\text { und Diät }\end{array}$ & $\begin{array}{c}\text { Progesteron } \\
\text { und Diät }\end{array}$ & $\begin{array}{l}\text { Östrogen } \\
\text { und } \\
\text { Progesteron }\end{array}$ & $\begin{array}{l}\text { Östrogen } \\
\text { und } \\
\text { Progesteron } \\
\text { und Diät }\end{array}$ \\
\hline $\begin{array}{l}{ }^{90} \mathrm{Sr}-\text { Gehalt in } \% \mathrm{~d} . \\
\text { inj. Dosis/Tier }\end{array}$ & 71,10 & 57,32 & 71,03 & 74,29 & 64,82 & 54,98 & 78,92 & 64,88 \\
\hline Körpergewicht [g] & 285 & 261 & 257 & 271 & 248 & 268 & 276 & 264 \\
\hline $\begin{array}{l}{ }^{90} \text { Sr-Gehalt in } \% \text { d. } \\
\text { inj. Dosis/Femur }\end{array}$ & 2,80 & 2,19 & 3,01 & 2,96 & 2,82 & 2,19 & 3,17 & 2,70 \\
\hline $\begin{array}{l}{ }^{90} \mathrm{Sr}-\mathrm{Konz} . \text { in } \% \mathrm{~d} . \\
\text { inj. Dosis } / 100 \mathrm{mg} \\
\text { Femurasche }\end{array}$ & 0,96 & 0,80 & 0,99 & 0,92 & 0,97 & 0,76 & 0,98 & 0,95 \\
\hline $\begin{array}{l}\text { Femuraschegewicht } \\
{[\mathrm{mg}]}\end{array}$ & 293 & 278 & 305 & 339 & 291 & 290 & 327 & 286 \\
\hline
\end{tabular}

Tab. 3. Untersuchungsergebnisse des Versuches II.

stimmungen der drei folgenden Meßgrößen, ${ }^{90} \mathrm{Sr}$ Gehalt, ${ }^{90} \mathrm{Sr}$-Konzentration des Femurs und das Femuraschegewicht, wurden 8 Wochen nach der Injektion des ${ }^{90} \mathrm{Sr}$ vorgenommen.

Die Wirkungen der Diät, des Östrogens und des Progesterons sind in Abb. 2 dargestellt. Die Verabreichung der Diät führte zu einer Verminderung des ${ }^{90} \mathrm{Sr}$-Gehaltes im Gesamttier um etwa 13\% der injizierten Dosis. Die Erniedrigung des Körpergewichts konnte nicht gesichert werden. Die Abnahme des ${ }^{90} \mathrm{Sr}$-Gehaltes und der ${ }^{90} \mathrm{Sr}$-Konzentration im Femur betrugen etwa $0,45 \%$ der injizierten Dosis bzw. 0,09\% der injizierten Dosis pro $100 \mathrm{mg}$ Femurasche. Der Einfluß auf das Femuraschegewicht bestand in einer Verminderung um 28 Milligramm.

Nach den Östrogengaben konnte eine Erhöhung des ${ }^{90} \mathrm{Sr}$-Gehaltes im Ganztier um etwa 6\% der injizierten Dosis festgestellt werden. Die Abnahme des Körpergewichts konnte nicht gesichert werden. Der ${ }^{90} \mathrm{Sr}$-Gehalt und die ${ }^{90} \mathrm{Sr}$-Konzentration des Femur waren um $0,35 \%$ der injizierten Dosis und $0,11 \%$ der injizierten Dosis pro $100 \mathrm{mg}$ Femurasche erhöht. Eine Wirkung auf das Femuraschegewicht war nicht nachweisbar.

Die Progesteronbehandlung führte zu einer Erhöhung des Femuraschegewichts um 18 Milligramm. Ein weiterer gesicherter Einfluß dieses Hormons auf die übrigen Meßgrößen konnte nicht beobachtet werden. Auch bei diesem Versuch bleiben nach kombinierter Anwendung der drei Behandlungsarten die Einzelwirkungen erhalten.

\footnotetext{
${ }^{6}$ H. Kriegel u. I. Kohl, Deutscher Röntgenkongreß, Teil B, Sonderbände zur Strahlentherapie, 62, 219 [1965].

7 W. LenkeIt, Sonderdruck aus : Züchtungskunde 31, 9 [1959].
}

\section{Besprechung der Ergebnisse}

Die Wirksamkeit der P-armen und Ca-reichen Diät auf die ${ }^{90} \mathrm{Sr}$-Dekorporation ist schon in früheren Versuchen untersucht worden und unsere Versuchsergebnisse stimmen mit deren Resultaten weiterhin überein ${ }^{3,4,6,8}$. Der starke Einfluß dieser Diät auf den Knochenstoffwechsel kommt darin zum Ausdruck, daß sowohl die Männchen als auch die männlichen Kastraten nach 9- bzw. 5-wöchentlicher auf die Diät folgende Normalfütterung ein noch deutlich niedrigeres Femuraschegewicht gegenüber den jeweiligen Kontrollen aufweisen. Die 6 Wochen p.i. festgestellte Körpergewichtsverminderung wird wohl durch das erniedrigte Knochengewicht und teilweise durch die schlechtere Aufnahme des Diätfutters während der 3-wöchigen Verabreichung verursacht sein.

Höhere Östrogendosen fördern die Verknöcherungsprozesse, verbunden mit einer verstärkten Retention von $\mathrm{Ca}$ und $\mathrm{P}$, und wirken wachstumshemmend ${ }^{1,9}$. Auch die ${ }^{90} \mathrm{Sr}$-Ausscheidung wird durch die Östrogenbehandlung, wie aus den Versuchsergebnissen zu ersehen ist, herabgesetzt.

Während das Femuraschegewicht 14 bzw. 8 Wochen p.i. keinen gesicherten Unterschied zur jeweiligen Kontrolle aufzeigt, war das Körpergewicht 6 Wochen p.i. noch deutlich erniedrigt. Genaueren Aufschluß über die Gewichtsveränderungen in diesem Zeitraum bei Versuchs- und Kontrolltieren gibt die Tab. 4. Hieraus ist zu ersehen, daß die Ver-

\footnotetext{
8 R. H. Wassermann u. C. H. Comar, Exp. Biol. Med. 103, 124 [1960].

9 J. Will, Zbl. Gynäkol., 82. Jahrgang, Heft 26, 973 [1960].
} 
Versuch I

\begin{tabular}{|c|c|c|c|c|}
\hline Zeit & $\begin{array}{l}\text { Kontr } \\
\text { Durchschn. } \\
\text { Gewicht } \\
{[\mathrm{g}]}\end{array}$ & $\begin{array}{l}\text { rolle } \\
\text { Gewichts- } \\
\text { zunahme } \\
\quad[\mathrm{g}]\end{array}$ & $\begin{array}{l}\text { Öst } \\
\text { Gewichts- } \\
\text { zunahme } \\
\text { [g] }\end{array}$ & $\begin{array}{l}\text { trogen } \\
\text { Durchschn. } \\
\text { Gewicht } \\
{[\mathrm{g}]}\end{array}$ \\
\hline${ }^{90} \mathrm{Sr}-\mathrm{Inj}$. & 139 & \multirow{4}{*}{$\begin{array}{l}+22 \\
+32 \\
+20\end{array}$} & \multirow{4}{*}{$\begin{array}{l}+10 \\
+13 \\
+9\end{array}$} & 137 \\
\hline 1 Wo. p. i. & 161 & & & 147 \\
\hline 2 Wo. p. i. & 193 & & & 161 \\
\hline 3 Wo. p. i. & 213 & & & 170 \\
\hline \multirow[t]{3}{*}{6 Wo. p. i. } & 262 & +49 & +65 & 235 \\
\hline & & +123 & +97 & \\
\hline & \multicolumn{4}{|c|}{ Versuch II } \\
\hline Zeit & \multicolumn{2}{|c|}{\begin{tabular}{cc}
\multicolumn{2}{c}{ Kontrolle } \\
Durchschn. Gewichts- \\
Gewicht & zunahme \\
[g] & {$[\mathrm{g}]$} \\
\end{tabular}} & \multicolumn{2}{|c|}{\begin{tabular}{cc}
\multicolumn{2}{c}{ Östrogen } \\
Gewichts- Durchschn. \\
zunahme & Gewicht \\
{$[\mathrm{g}]$} & {$[\mathrm{g}]$}
\end{tabular}} \\
\hline${ }^{90} \mathrm{Sr}-\mathrm{Inj}$. & 215 & \multirow{3}{*}{$\begin{array}{l}+8 \\
+13 \\
+15\end{array}$} & \multirow{3}{*}{$\begin{array}{l}-2 \\
-5 \\
+4\end{array}$} & 211 \\
\hline 1 Wo. p. i. & 223 & & & 209 \\
\hline $\begin{array}{l}2 \text { Wo. p. i. } \\
3 \text { Wo. p. i. }\end{array}$ & $\begin{array}{l}236 \\
251\end{array}$ & & & $\begin{array}{l}204 \\
208\end{array}$ \\
\hline \multirow[t]{2}{*}{6 Wo. p. i. } & 285 & +34 & +49 & 257 \\
\hline & & +70 & +46 & \\
\hline
\end{tabular}

Tab. 4. Gewichtsveränderungen bei östrogenbehandelten Ratten und Kontrollen. suchstiere während der Östrogenbehandlung einer auffallenden Wachstumshemmung unterliegen. Nach Abschluß der Hormongaben findet eine gewisse Kompensation durch verstärkte Gewichtszunahme bei den Versuchstieren statt. Sie erreichen jedoch 3 Wochen nach Beendigung der Östrogenverabreichung noch nicht das Gewicht der Kontrolltiere.

Nach Verabreichung von Progesteron blieben das Körpergewicht und die ${ }^{90} \mathrm{Sr}$-Ausscheidung im Vergleich zu den Kontrollen unbeeinflußt. Interessant ist jedoch die Erhöhung des Knochenaschegewichts bei den Kastraten. Auf Grund der positiven Stoffwechselbilanz während der Gestation bei Säugetieren tritt eine Knochenaschegewichts-Zunahme ein ${ }^{7}$. Wahrscheinlich haben wir bei den Kastraten durch das Gelbkörperhormon einen gestationsähnlichen Stoffwechselzustand geschaffen, der sich in dem erhöhten Aschegewicht ausdrückt. Durch die Erhöhung des Knochenaschegewichts wäre eine Erniedrigung der ${ }^{90} \mathrm{Sr}$-Konzentration zu erwarten. Die wenig ausgeprägte Konzentrations-Erniedrigung konnte jedoch statistisch nicht gesichert werden.

Herrn Prof. Dr. Dr. H. Langendorff sei für wertvolle Hinweise herzlich gedankt. Für ihre ausgezeichnete Mitarbeit sind wir Frl. Verena Bühler zu Dank verpflichtet. 